\title{
Overwhelmed healthcare services and the prevailing threat of COVID-19 infection among healthcare workers: Implications on dermatology residency programs
}

Shivam Goyal, Shibani Bhatia, Smitha Prabhu

Department of Dermatology, Kasturba Medical College, Manipal Academy of Higher Education (MAHE), Manipal, India

Corresponding author: Dr. Smitha Prabhu, E-mail: smitha.prabhu@manipal.edu

Sir,

The coronavirus disease 2019 (COVID-19) pandemic has now restricted hospital services to emergency care. The cutback of routine patients to the department of dermatology has aimed to reduce the exposure to healthcare workers (HCW) and transmission of infection from infected HCWs to patients.

COVID-19 inpatients with skin manifestations are being evaluated in a limited manner by dermatologists based on images. Dermatologic diagnosis relies heavily on visual aid, especially a closer view of up to $20 \mathrm{~cm}$ for diagnosis along with relevant clinical history taking. The limitation of consultations has severely impacted case-based and bedside learning for dermatology residents. Teledermatology facilities may be utilized for distant and close-up views of lesions and corroboration with relevant medical history taking to overcome this loss of learning experience [1].

Procedural dermatology has also taken a hit as most procedures, such as thread lifts, fillers, Botox injections, chemical peels, and laser procedures, involve the face and close contact with the patient; therefore, it has been put on hold. In the meantime, residents can use online training modules available on their dermatology society's website to hone their skills on existing and newer procedures.

Dermoscopy, which relies on even closer contact with the patient, has been discontinued indefinitely by the advice of society guidelines. Precautionary measures in performing dermoscopy include wearing protective equipment by both the patient and the doctor, disinfecting the lesional areas and the dermatoscope with $70 \%$ isopropyl alcohol for one minute before and after its use, and preferring noncontact ways of dermoscopy [2].

There is a risk of developing sudden respiratory distress among asymptomatic patients in dermatology wards. Thus, residents should be able to respond to such emergency conditions before the arrival of emergency teams [3]. A short-duration online refresher course designed by a pulmonologist can help to meet the sudden need for emergency training and to provide crucial first response to patients before the arrival of the emergency and pulmonary care team.

Residents are now required to wear personal protective equipment (PPE), which are known to cause cutaneous damage after long hours of use such as erosions, ulcers, and indentations, as well as exacerbate other skin conditions, such as acne and seborrheic dermatitis [4]. Gloves of all three types-latex, nitrile, and plastic, especially the powdered varieties—have been associated with contact dermatitis. Skincare regimen, such as moisturizing before using protective equipment, using face shields instead of goggles, preferring powderfree gloves, and washing hands after using gloves, are some recommendations that can help in keeping the skin of HCWs healthy [5,6]. Counseling programs on cutaneous damage due to PPE are a useful aid as well.

\footnotetext{
How to cite this article: Goyal S, Bhatia S, Prabhu S. Overwhelmed healthcare services and the prevailing threat of COVID-19 infection among healthcare workers: Implications on dermatology residency programs. Our Dermatol Online. 2020;11(Supp. 2):31-32.

Submission: 06.08.2020; Acceptance: 24.08.2020

DOI: 10.7241 /ourd.2020S2.12
} 
Lastly, the COVID-19 pandemic has generated fear among HCWs across all specialties, considering the deaths of several frontline HCWs in nations such as China, Italy, and the U.S. Dermatology residents are working toward screening and sample collection, management of inpatients with influenza-like illness (ILI) and with skin diseases, and management of confirmed COVID-19 inpatients. The looming threat of infection and a subsequent possible life-threatening situation and increased risk of exposure to loved ones, coupled with a global shortage of PPE have led to states of panic, anxiety, and uncertainty among residents [7] . Social media platforms working toward mental support and psychological help can help to address the mental health issues of HCWs working on the frontline.

In conclusion, COVID-19 has changed dermatology residency to a great extent and, since the medical literature on COVID-19 is evolving continuously, teaching methodologies should evolve simultaneously. All failure is failure to adapt; all success is successful adaptation.

\section{Consent}

The examination of the patient was conducted according to the principles of the Declaration of Helsinki.

\section{REFERENCES}

1. Lee I, Kovarik C, Tejasvi T, Pizarro M, Lipoff JB. Telehealth: Helping your patients and practice survive and thrive during the COVID-19 crisis with rapid quality implementation. J Am Acad Dermatol. 2020;82:1213-4.

2. Jakhar D, Kaur I, Kaul S. Art of performing dermoscopy during the times of coronavirus disease (COVID-19): simple change in approach can save the day! J Eur Acad Dermatol Venereol. 2020;34:e242-4.

3. Tao J, Song Z, Yang L, Huang C, Feng A, Man X. Emergency management for preventing and controlling nosocomial infection of the 2019 novel coronavirus: implications for the dermatology department. Br J Dermatol. 2020;182:1477-8.

4. Zhang B, Zhai R, Ma L. 2019 novel coronavirus disease epidemic: skin protection for healthcare workers must not be ignored. J Eur Acad Dermatol Venereol. 2020 May 2;10.1111/jdv.16573.

5. Tabary M, Araghi F, Nasiri S, Dadkhahfar S. Dealing with skin reactions to gloves during the COVID-19 pandemic. Infect Control Hosp Epidemiol. 2020 May 8;1-2. doi: 10.1017/ice.2020.212.

6. Zheng Y, Lai W. Dermatology staff participate in fight against Covid-19 in China. J Eur Acad Dermatol Venereol. 2020;34:e210-1.

7. Goldust M, Agarwal K, Kroumpouzos G, Jafferany M, Lotti T, Podder I. Mental health status of dermatologists during the COVID-19 pandemic: A technology-based therapy. Dermatol Ther. 2020 May 31;e13723. doi: 10.1111/dth.13723.

Copyright by Shivam Goyal, et al. This is an open-access article distributed under the terms of the Creative Commons Attribution License, which permits unrestricted use, distribution, and reproduction in any medium, provided the original author and source are credited. Source of Support: Nil, Conflict of Interest: None declared. 